\title{
Characterization of liquid crystal on silicon-SLMs for in-situ laser beam steering to enable RAMAN imaging
}

Sebastian Ritter, Sven Augustin, Anko Börner, David Krutz, Ute Böttger

Sebastian Ritter, Sven Augustin, Anko Börner, David Krutz, Ute Böttger, "Characterization of liquid crystal on silicon-SLMs for in-situ laser beam steering to enable RAMAN imaging," Proc. SPIE 11852, International Conference on Space Optics - ICSO 2020, 118526E (11 June 2021); doi: $10.1117 / 12.2600289$

SPIE Event: International Conference on Space Optics - ICSO 2021, 2021, Online Only 


\section{International Conference on Space Optics-ICSO 2020}

Virtual Conference

30 March-2 April 2021

Edited by Bruno Cugny, Zoran Sodnik, and Nikos Karafolas
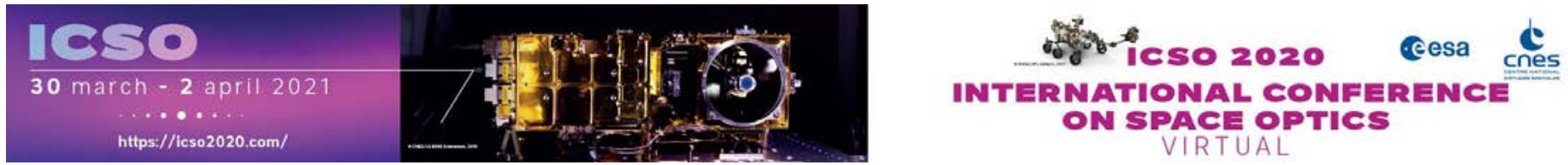

\section{Characterization of Liquid Crystal on Silicon-SLMs for In-Situ Laser Beam Steering to enable RAMAN Imaging}

\section{Cesa isoporecetings ecnes}




\title{
Characterization of Liquid Crystal on Silicon-SLMs for In-Situ Laser Beam Steering to enable RAMAN Imaging
}

\author{
Sebastian Ritter ${ }^{\mathrm{a}}$, Sven Augustin ${ }^{\mathrm{ab}}$, Anko Börner ${ }^{\mathrm{a}}$, David Krutz ${ }^{\mathrm{a}}$, and Ute Böttger ${ }^{\mathrm{a}}$

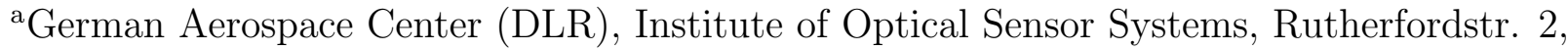 \\ 12489 Berlin, Germany \\ ${ }^{\mathrm{b}}$ Humboldt University of Berlin, Department of Physics, Newtonstr. 15, 12489 Berlin, \\ Germany
}

\begin{abstract}
In the future, the use of lunar regolith for resource extraction and manufacturing of construction materials will play a significant role in lunar exploration projects. Raman spectroscopy can contribute to the quality control of materials made of moon regolith. To improve system reliability in Raman point mapping it is proposed to use dynamic light shaping to reduce the risk of thermal and mechanical damage. An approach to characterize a Liquid Crystal on Silicon (LCoS) Spatial Light Modulator (SLM) is shown. For this purpose, influence parameters are identified and evaluated with the help of a laboratory setup and a complementary simulation in order to evaluate the possibilities and limitations of Raman raster point mapping in respect to the deflection and spot shaping.
\end{abstract}

K eywords: Spatial Light Modulator, Liquid Crystal on Silicon, LCoS, Raman Imaging, Space Application

\section{INTRODUCTION}

To support future space exploration In-Situ Resource Utilization (ISRU) especially the processing of lunar regolith is becoming more and more significant. For example, with the help of printed or sintered lunar regolith components, lunar stations can be expanded [1], or raw materials can be extracted [2]. Possible processing of lunar regolith with selective laser melting is contributed in [3] and the moonrise project [4].

Raman spectroscopy is proposed for the quality assessment of sintered and 3D-printed lunar regolith and lunar regolith simulant elements to investigate the silica-based glass content and to identify minerals on surfaces [5-7]. Raman spectroscopy is an optical spectroscopic technique to inspect inner molecular frequency modes excited by laser light to determine material characteristics e.g. for minerals.

To apply this method of quality assurance directly on the lunar surface, a Raman spectrometer such as the RAX [8] by the German Aerospace Center (DLR) selected for MartianMoons eXploration (MMX) [9] mission by the Japan Aerospace Exploration Agency JAXA is envisaged with an extension. This will allow a single point measurement to be extended to a point-by-point mapping of a sample area to obtain a spatially resolved image. Raman imaging combines spatial resolution and spectral data to evaluate changes in material properties over an area, and is therefore well suited for quality control of manufactured components.

The utilization of a Spatial Light Modulator (SLM) specifically a Liquid Crystal on Silicon SLM (LCoS) instead of a mechanical scanner (e.g. used in the SHERLOC [10] instrument on board of Mars 2020 mission) reduces the risk of mechanical failure, vulnerability to vibration and temperature variation at the instrument level and also enables on-mission adjustments of optical parameters of a laser spectrometer.

An LCoS is a reflective liquid crystal microdisplay that is able to shift the phase of coherent light pixel-wise and acts this way as a spatial light modulator. It is often utilized for applications for real-time holography,

Further author information: Sebastian Ritter E-mail: Se.Ritter@dlr.de 
diffractive optics and also in image and video display applications. An LCOS device can be either transmissive or reflective and can be used to alter the polarization or the phase of an incident coherent light beam [11]. The reflective LCoS are composed of a silicon complementary metal-oxide semiconductor (CMOS) backplane, which controls the liquid crystal layer's light modulating properties [12].

A wavefront from a collimated continuous wave laser, used for Raman spectroscopy, is straightforwardly guided by diffractive patterns projected on the LCoS onto a sample of interest to dynamically adjust the focus and deflection of the laser. The accuracy of the laser focusing and steering depends on quantization effects, caused by various parameters of the SLM, like the given number of pixels.

In [13], LCoS elements were shown to be suitable for space applications and their capabilities for potential space qualification were demonstrated.

\section{EXPERIMENTAL SETUP}

The experimental setup is shown in Fig. 1. The used laser wavelength of $532 \mathrm{~nm}$ is based on the application Raman imaging. A fiber-coupled laser is collimated using a fiber collimator and guided perpendicularly to the LCoS via a beam splitter (50/50 to gain maximum laser power on the sample plane). A beam splitter is employed to not modify the modulation properties by incident angles. So, no further optimization steps need to be performed to correct these effects [14]. The light is reflected from the active surface of the LCoS and can be dynamically focused and steered onto a sample for Raman imaging. At this point, the sample area, there is a camera (charge-coupled device (CCD) $3.75 \mu \mathrm{m}$ pixel grid) for evaluation of the laser spot.

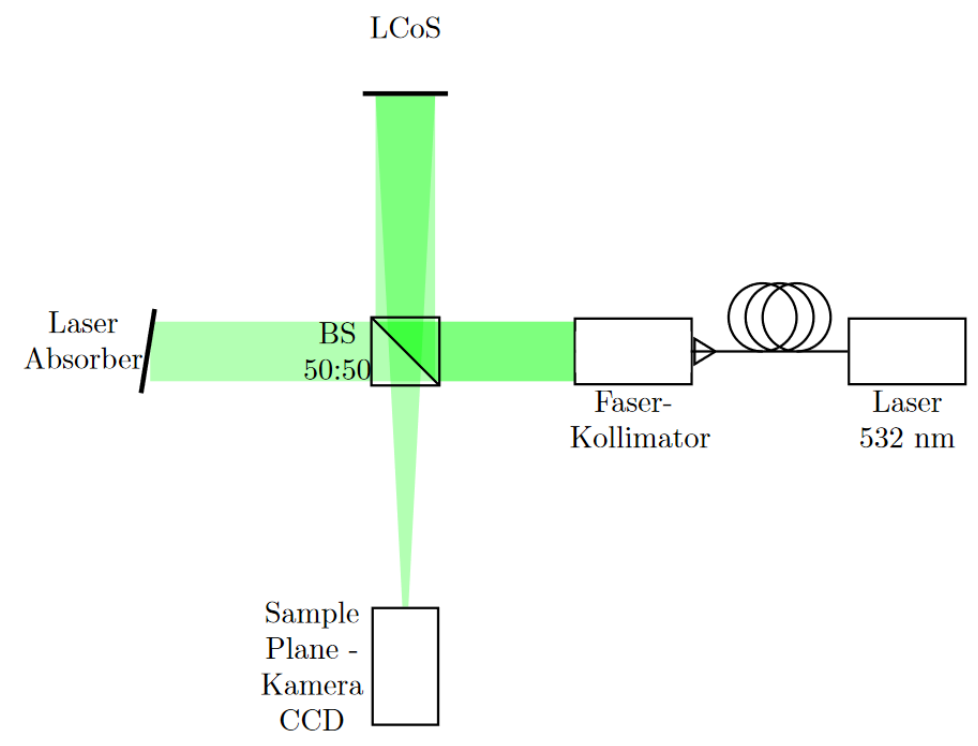

Figure 1. LCoS point mapping characterization setup

For the characterization the parallel aligned nematic liquid crystal display Pluto-2 from Holoeye is used, which operates as phase only LCoS with reflective backside. In operation the device is controlled with 8 bits (256 levels) addressing from a PC as green monitor channel [15]. The relevant properties for this work are listed in Tab. 1. 
Table 1. LCoS properties [15] for definitions see [16]

\begin{tabular}{|l|l|}
\hline Resolution & $1920 \times 1080$ \\
\hline Pixel Pitch & $8.0 \mu \mathrm{m}$ \\
\hline Fill Factor & $93 \%$ \\
\hline Active Area & $15.36 \times 8.64 \mathrm{~mm}\left(0.7^{\prime \prime}\right.$ Diagonal) \\
\hline Dynamic Range (Phase Levels) & 8 Bit $(256)$ \\
\hline Phase Resolution & $0.0137 \pi /$ Level \\
\hline Maximum Phase Modulation $(\mathrm{rad})$ & $3.5 \pi$ @ $530 \mathrm{~nm}$ \\
\hline Wavelength Range & 500 to $670 \mathrm{~nm}$ \\
\hline Light Utilization Efficiency & $94 \%$ \\
\hline Screen Refreshing Frequency & $60 \mathrm{~Hz}$ \\
\hline
\end{tabular}

\section{CHARACTERIZATION CONCEPT}

The main goal is to transfer the characterization setup to a simulation representation to model the optical properties of the LCoS in relation to the Raman point mapping application. To achieve this goal, the laboratory setup Fig. 1 was created and the following measurements were performed.

Subsequently, the setup is modeled component by component, and using the Angular Spectrum Method (ASM) a light field is propagated. This way characteristics of laser and components (e.g. beam splitter) can be simulated.

\subsection{Laser Spot Generation}

To achieve the focusing of the laser, a Fresnel zone plate is applied to the active surface of the LCoS. For this purpose, in contrast to the classical fresnel zone plate, which is represented in binary, the 256 phase steps are used, which are provided by the LCoS. When using the zone plate, the $1^{\text {st }}$ diffraction order forms the intended focused point, while the other diffraction orders, including the undiffracted portion, contribute to noise and reduce the focusing efficiency of the configuration. The focal length is determined by Eq. 1 [15], where $\mathrm{N}$ is the inner radius of the Fresnel zonal plate, $\mathrm{p}$ is the pixel pitch (here $8 \mu \mathrm{m}), \lambda$ is the wavelength and $\mathrm{f}$ is the focal length and equals the distance between the SLM and the sample.

$$
f=\frac{(N * p)^{2}}{2 \lambda}
$$

For the deflection of the laser beam, in order to map a sample point by point, the zone plate is superimposed with a blazed grating. This blazing grating is constructed of periodic sawtooth phase ramps inclined in the direction of the desired deflection. This superposition manipulates the angle between the undiffracted part of the light $\left(0^{t h}\right.$ order) and the intended focus spot $\left(1^{s t}\right.$ order). Thus, a laser can be adjusted in 3D space, by setting the zone plate and grating parameters. It is also possible to expand the size of the laser spot with the LCoS. The laser spot size is an important parameter in Raman spectroscopy, affecting the spatial resolution and the signal to noise ratio.

\subsection{Process of System Design}

For the investigation of the laser spot to be generated, it must first be defined from the application which diameter is suitable for the application. This can be derived from the area coverage of sampling and on the other hand from signal-to-noise ratio (SNR) analyses from existing Raman configurations. From reference measurements taken on relevant regolith simulant, the required spectroscopic parameters such as optical bandwidth, spectral resolution and SNR can be determined. These investigations are considered as input data for this work. After that, the properties of focusing and deflection are determined with the laboratory setup Fig. 1. Finally, the setup can be simulated with a wavefront simulation. 


\subsection{Challenges}

The device-specific parameters of the LCoS, which influence the optical performance for the application, must be identified and their effect modeled. These include, for example, the spatial resolution of the LCoS, the reduction of which harms the focusing performance. Further properties under investigation are the number of steps (dynamics) of the phase shift and pixel size. Understanding these effects will play an important role in modeling and their influence can be evaluated.

Likewise, the other unwanted diffraction orders of the zone plate and the grid structure of the LCOS display itself can be compared to the $1^{\text {st }}$ order (intended focus spot).

For an evaluation of the influence of degradation damage caused by energetic radiation under space conditions, the failure of pixels or portions of the active area of the LCoS are as well considered as the uniform change of the reflexion properties.

\subsection{M easurements}

In order to evaluate the setup characteristics, raster scans of the beam were performed at significant locations. This allows the entry side of the LCoS to be monitored. For this purpose, a CCD camera $3.75 \mu \mathrm{m}$ pixel pitch is employed. In the sample/camera plane, a raster scan is also performed to evaluate the beam profile with the LCoS switched off. Diffraction artifacts through the edges of the active area are identifiable. A rasterscan with an employed Fresnel zone plate shows the intensity distribution between the diffraction orders of the focus, the $2^{\text {nd }}$ order and the undiffracted part (Fig. 3a).

The focusing power is measured at the focal point at different focal lengths and at fixed focal length along the propagation axis Fig. 2a. Spot sizes are measured in both major axes using the $4 \mathrm{D} \sigma$ method and acquired as a profile. The evaluation plot can be found in Fig. 2b. The achievable deflection angles and the corresponding spot sizes can be determined in the camera plane by gradually overlaying the zone plate with the smallest possible increments of diffraction grating that the LCoS accepts. This examination is performed in both possible dimensions.

A reduction of the spatial resolution at the LCoS (from originally $1920 \times 1080$ pixel) and the reduction of the possible phase steps (from originally 256 possible steps) can demonstrate a reduction of the focusing power.

To simulate radiation induced degradation, different numbers of random pixels are switched off from a zone plate and the focusing power is evaluated.

\subsection{Wavefront Simulation}

The simulation aims to reproduce the illumination conditions of the LCoS and the effect of the LCoS on the sample plane. For this purpose, the monochromatic Angular Spectrum Method (ASM) is applied.

The ASM is a technique to model the propagation of a wavefront from one plane to another through a homogeneous medium. The angular spectrum representation is found to be a very powerful method for the description of laser beam propagation and light focusing [17].

A light field at level 0 is decomposed into its wave components by a $2 \mathrm{D}$ spatial Fourier transform. The individual wave components are then propagated to level 1 using the Helmholz wave equation with additional constraints. Here, the result from the frequency domain is calculated back into the spatial frequency domain using the inverse Fourier transform function [18]. 


\section{RESULTS}

Fig. 2a shows the average diameter of laser spots of about $25-80 \mu \mathrm{m}$ when sampling along the propagation axis in focus and its nearby area. 0 marks the focal plane at a focal length of $155 \mathrm{~mm}$. The measurements are executed with $0.1 \mathrm{~mm}$ resolution in a range of $4 \mathrm{~mm}$ around the focus plane. The values represent the entire setup. Fig. $2 \mathrm{~b}$ shows the evaluation overview for the beam measurement. The main axes of the spot (blue and yellow axis) were detected and along these, the diameters were determined with the $4 \mathrm{D} \sigma$ method. The line plots along the main axes are compared with a gaussian fit. The diameter range is shown as blue dashed lines in the plots. This method was chosen because the outer regions of the spot are given special consideration. It is also recommended as ISO standard method for divergence angles and beam propagation ratios of laser beams. A CCD camera (Allied Vision Prosilica GC1290C) with a $3.75 \mu \mathrm{m}$ pixel grid was used.

A raster scan was performed in the sample plane Fig. 3a to evaluate the focusing efficiency and the distribution over the different orders and undiffracted fractions. The summed intensity of the focus spot to undiffracted residual light and the intensity sum of the further orders was determined. Relative to the summed total intensity, a summed intensity of $75.3 \%$ was determined for the $1^{\text {st }}$ order and a summed intensity of $7.3 \%$ for the further orders. These ratios reflect the overall performance of the setup including the use of the LCoS and the zone plates inherent characteristics.

For a simulated degradation of random pixels due to radiation, Fresnel zone plates are manipulated with an overlay of a number of randomly distributed pixels with a phase shift of $0 \pi$. Only deviations within the statistically distributed measurement results were found in the range up to $8 \%$ dead pixels. For a failure of 400000 pixels, $30 \%$ of the total amount of LCoS (at $1920 \times 1080$ pixels), it was found that a spot was enlarged by $5.8 \%$ in diameter at a focal length of $155 \mathrm{~mm}$, but with a significant drop in intensity.

For the simulated propagation from the LCoS area to the sample plane Fig. 3c,d, a Fresnel zone plate with 155 $\mathrm{mm}$ focal length was applied and a collimated laser with $532 \mathrm{~nm}$ modeled with superimposed Zernike patterns were used as phase input. Included were diffraction effects around the edges of the active area, which act as an aperture. The diffraction order angles can be reproduced, the exact spot geometry is still in ongoing development.

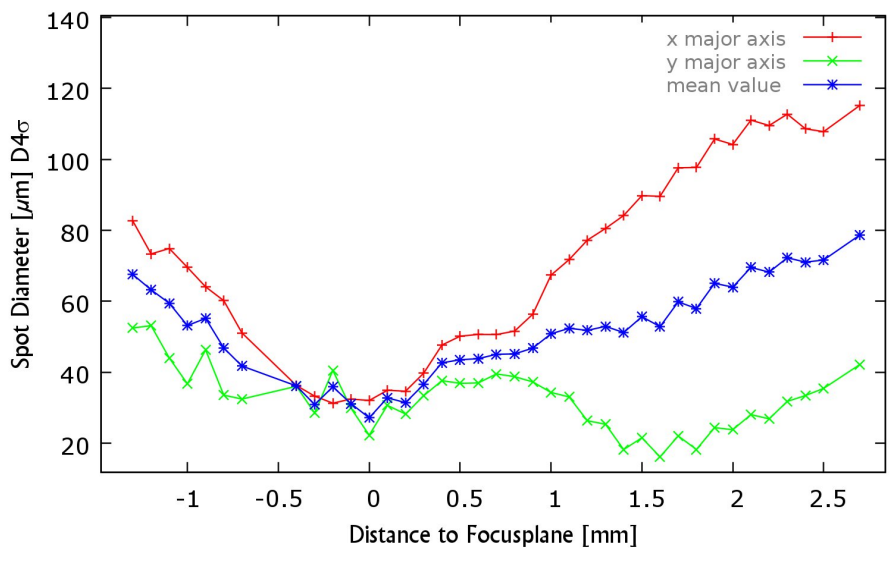

(a)
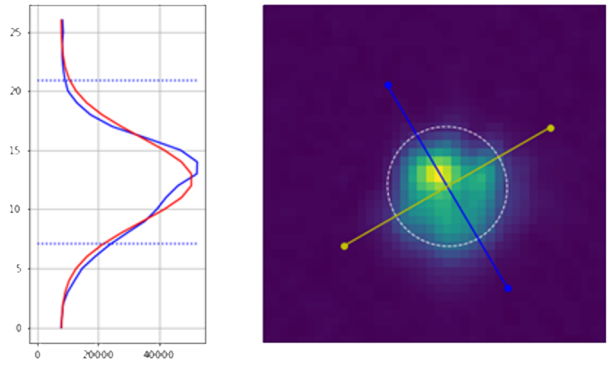

pixel size: $3,75 \mu \mathrm{m}$ D4sigma waist $x: 53,37 \mu \mathrm{m}$ waist y $51,95 \mu \mathrm{m}$

Gaussian fit

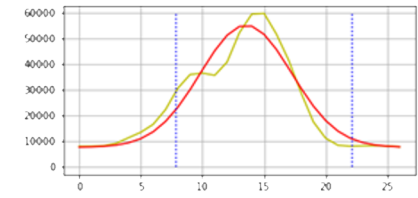

(b)

Figure 2. Analysis of focused beam a) spot size along the propagation axis b) profile in focus (D4 $\sigma$ Method) 


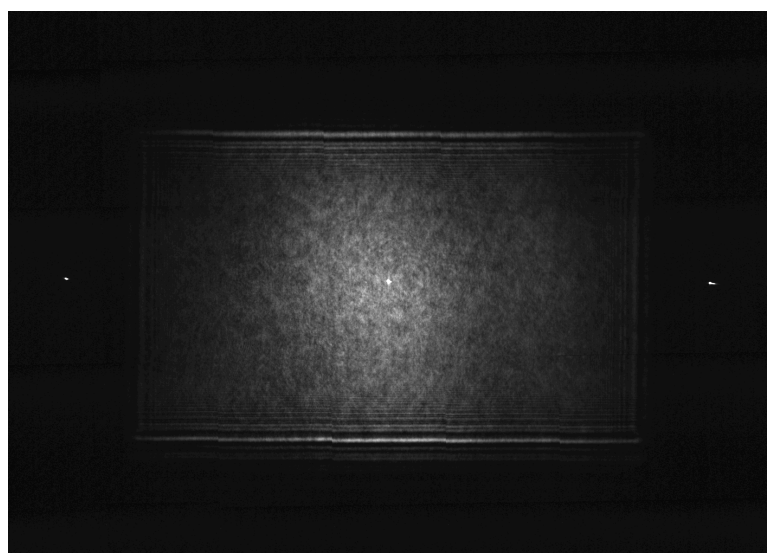

(a)

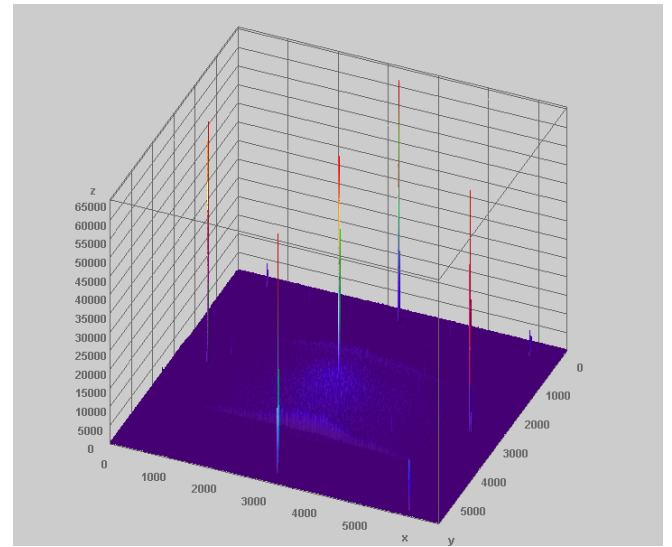

(c)

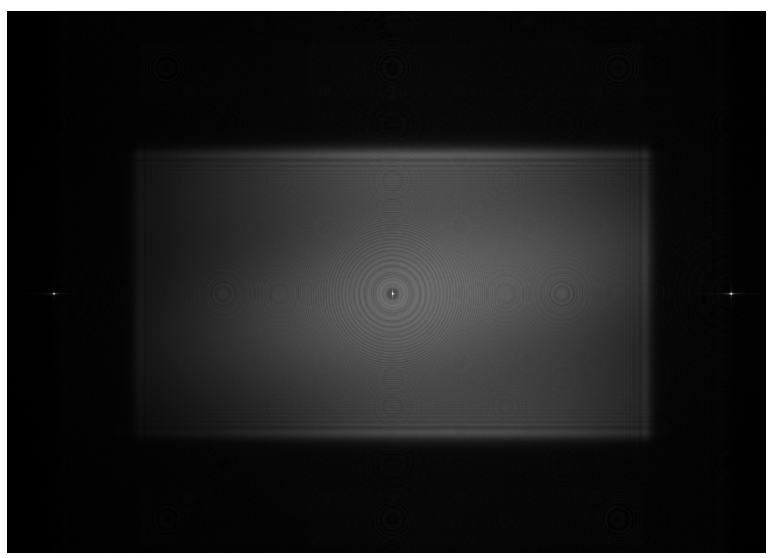

(b)

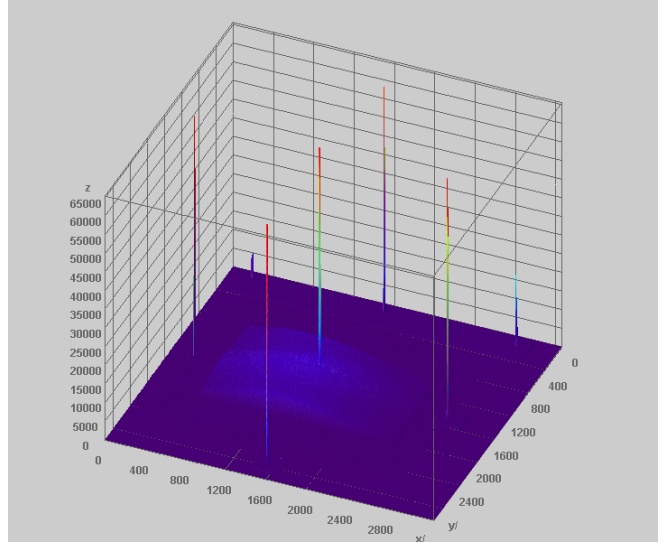

(d)

Figure 3. Wavefront in sample plane, spikes mark the intended focus and other diffraction orders a) raser scan b) wavefront propagation simulation (Angular Spectrum Method) c) raster scan 3D representation d) simulation 3D representation

\section{DISCUSSION}

The preliminary results presented here show that the LCoS in combination with zone plates is suitable to focus a laser beam at different focal lengths and deflections and also with which dependencies. The spot diameters depend, among other things, on the focal length. For distracted beams, the focal length expands by a small amount, so the spot diameter can be statistically determined as a dependence of the deflection. For the simulation, both the focusing with further diffraction orders and the deflection can be simulated in a good approximation.

\section{CONCLUSIONS}

It can be shown that a laser spot can be dynamically adapted to the requirements of Raman spectroscopy and point mapping using an LCoS. By examining the spot characteristics at different focal lengths and deflections, two-dimensional scanning can be optimized in terms of measuring distance, area coverage and taking Raman spectroscopy into account also the integration time and SNR. In addition to Fresnel zone plates, it is possible to shape the laser beam with computer-generated phase patterns to compensate for inhomogeneities in the beam characteristics or to shape the beam into various geometries, such as multiple foci, which would enable other sampling methods. 


\section{REFERENCES}

[1] Cesaretti, G., Dini, E., Kestelier, X. D., Colla, V., and Pambaguian, L., "Building components for an outpost on the lunar soil by means of a novel 3d printing technology," Acta Astronautica 93, 430-450 (jan 2014).

[2] Baasch, J., Windisch, L., Koch, F., Linke, S., Stoll, E., and Schilde, C., "Regolith as substitute mold material for aluminum casting on the moon," Acta Astronautica 182, 1-12 (may 2021).

[3] Gerdes, N., Fokken, L. G., Linke, S., Kaierle, S., Suttmann, O., Hermsdorf, J., Stoll, E., and Trentlage, C., "Selective laser melting for processing of regolith in support of a lunar base," Journal of Laser Applications 30, 032018 (aug 2018).

[4] Lotz, C., Gerdes, N., Sperling, R., Lazar, S., Linke, S., Neumann, J., Stoll, E., Ertmer, W., and Overmeyer, L., "Tests of additive manufacturing and other processes under space gravity conditions in the einsteinelevator," Volume 2020 , Issue 12 (2020).

[5] Robens, E., Dabrowski, A., Mendyk, E., Goworek, J., Skrzypiec, K., Drewniak, M., Dumańska-Słowik, M., Gac, W., Dobrowolski, R., Pasieczna-Patkowska, S., Huber, M., Iwan, M., Kurzydłowski, K., Płociński, T., Ryczkowski, J., Rzaczyńska, Z., and Sobczak, J., "Investigation of surface properties of lunar regolith - part IV," Annales UMCS, Chemistry 63 (jan 2008).

[6] Wang, A., Jolliff, B. L., and Haskin, L. A., "Raman spectroscopy as a method for mineral identification on lunar robotic exploration missions," Journal of Geophysical Research 100(E10), 21189 (1995).

[7] Tuschel, D., "Why are the raman spectra of crystalline and amorphous solids different?," 32, $26-33$ (03 2017).

[8] Hagelschuer, T., Böttger, U., Buder, M., et al., "Rax - a raman spectrometer for mmx onboard the rover for phobos," in [13th IAA Low-Cost Planetary Missions Conference], 13th IAA Low-Cost Planetary Missions Conference (June 2019).

[9] Kuramoto, K., Kawa-katsu, Y., Fujimoto, M., Genda, H., Imamura, T., Kameda, S., Matsumoto, K., Miyamoto, H., Morota, T., Na-gaoka, H., Nakamura, T., Ogawa, K., Otake, H., Ozaki, M., Sasaki, S., Senshu, H., Tachibana, S., Terada, N., Usui, T., Wada, K., Watanabe, S., and study team, M., "Martian moons exploration (mmx) conceptual study update.," in [49 th Lunar and Planetary Science Conference (LPIContrib.No.2083)], (2018).

[10] Beegle, L., Bhartia, R., and and, M. W., "Sherloc: Scanning habitable environments with raman luminescence for organics chemicals," (mar 2015).

[11] Zhang, Z., You, Z., and Chu, D., "Fundamentals of phase-only liquid crystal on silicon (LCOS) devices," Light: Science 83 Applications 3, e213-e213 (oct 2014).

[12] Márquez, A. and Lizana, Á., "Special issue on liquid crystal on silicon devices: Modeling and advanced spatial light modulation applications," Applied Sciences 9, 3049 (jul 2019).

[13] Silva-López, M., Campos-Jara, A., and Álvarez-Herrero, A., "Validation of a spatial light modulator for space applications," in [International Conference on Space Optics - ICSO 2018], Karafolas, N., Sodnik, Z., and Cugny, B., eds., SPIE (jul 2019).

[14] Lizana, A., Martín, N., Estapé, M., Fernández, E., Moreno, I., Márquez, A., Iemmi, C., Campos, J., and Yzuel, M. J., "Influence of the incident angle in the performance of liquid crystal on silicon displays," Optics Express 17, 8491 (may 2009).

[15] Holoeye, [Pluto-2 Phase Only Spatial Light Modulators], Holoeye (2020).

[16] Holoeye, "https://holoeye.com/spatial-light-modulators/," (Feb. 2021).

[17] Lukas Novotny, B. H., [Principles of Nano-Optics], Cambridge University Press (Sept. 2012).

[18] Goodman, J., [Introduction to Fourier optics], McGraw-Hill, New York (1996). 